\title{
Investigating the scalar sector of left-right symmetric models with leptonic probes
}

\author{
Debasish Borah,,$^{1, *}$ Benjamin Fuks, ${ }^{2,3, \dagger}$ Deepanjali Goswami, ${ }^{1,+}$ and P. Poulose ${ }^{1, \S}$ \\ ${ }^{1}$ Department of Physics, Indian Institute of Technology Guwahati, Assam 781039, India \\ ${ }^{2}$ Sorbonne Université, CNRS, Laboratoire de Physique Théorique et Hautes Énergies, \\ LPTHE, F-75005 Paris, France \\ ${ }^{3}$ Institut Universitaire de France, 103 boulevard Saint-Michel, 75005 Paris, France
}

(Received 24 May 2018; published 7 August 2018)

\begin{abstract}
We investigate the potential collider signatures of singly charged and doubly charged Higgs bosons such as those arising in minimal left-right symmetric models. Focusing on multileptonic probes in the context of the high-luminosity run of the Large Hadron Collider, we separately assess the advantages of the fourleptonic and trileptonic final states for a representative benchmark setup designed by considering a large set of experimental constraints. Our study establishes possibilities of identifying singly charged and doubly charged scalars at the Large Hadron Collider with a large significance, for luminosity goals expected to be reached during the high-luminosity phase of the Large Hadron Collider. We generalize our results and demonstrate that existing limits can in principle be pushed much further in the heavy mass regime.
\end{abstract}

DOI: $10.1103 /$ PhysRevD.98.035008

\section{INTRODUCTION}

The Standard Model of particle physics has been established as the most successful theory describing the elementary particles and their interactions (with the exception of gravity), especially after the discovery of a Standard-Modellike Higgs boson at the Large Hadron Collider (LHC) in 2012 [1,2]. Despite its successes, the Standard Model features several conceptual issues and practical limitations that have motivated the particle physics community to focus on theoretical developments and experimental studies of physics beyond it. In particular, the Standard Model cannot provide any explanation for the observed nonzero neutrino masses and mixings [3]. In the Standard Model, the Higgs field is responsible for the generation of the masses of all known fundamental particles, but it cannot accommodate the tiny observed neutrino masses within only renormalizable interactions. The situation nonetheless changes at the nonrenormalizable level since neutrino masses can be generated through the dimension-five Weinberg operator [4] that generally arises, within a renormalizable ultravioletcomplete theory where new heavy fields are introduced,

\footnotetext{
*dborah@iitg.ac.in

fuks@1pthe.jussieu.fr

${ }^{\ddagger}$ g.deepanjali@iitg.ac.in

poulose@iitg.ac.in
}

Published by the American Physical Society under the terms of the Creative Commons Attribution 4.0 International license. Further distribution of this work must maintain attribution to the author(s) and the published article's title, journal citation, and DOI. Funded by SCOAP. through a seesaw mechanism. The different realizations of such a mechanism can be broadly classified into three categories named type I [5-9] (that relies only on righthanded neutrinos coupling to the Higgs field), type II [10-13] [that makes use of a new scalar field lying in the adjoint representation of $S U(2)_{L}$ ], and type III [14] [where at least two extra fermionic fields lying in the adjoint representation of $S U(2)_{L}$ are included] seesaws.

In most common seesaw implementations, heavy fields are supplemented to the Standard Model in an ad hoc fashion so that the desired neutrino properties are reproduced after the breaking of the electroweak symmetry. Seesaw options where the symmetries of the Standard Model are extended also exist, as in left-right symmetric theories [15-26] where minimal and nonminimal realizations naturally feature type I/II and type III seesaw mechanisms, respectively. In the left-right symmetric paradigm, the Abelian hypercharge symmetry group of the Standard Model $U(1)_{Y}$ is extended to an $S U(2)_{R} \times$ $U(1)_{B-L}$ gauge group and the right-handed $S U(2)_{L}$ fermionic singlets are collected into $S U(2)_{R}$ doublets, which naturally requires the introduction of right-handed neutrino fields in the spectrum. Focusing on minimal model building possibilities (and thus on type I and type II seesaws), the extended gauge symmetry is spontaneously broken down to the Standard Model gauge symmetry thanks to a scalar field that lies in the adjoint representation of $S U(2)_{R}$ or $S U(2)_{L}$ for a type I and type II seesaw mechanism, respectively. This field acquires a nonvanishing vacuum expectation value that yields neutrino mass generation. In their most minimalistic form, left-right symmetric theories 
are moreover symmetric under parity transformations in the ultraviolet regime, although low-energy parity violation arises after the spontaneous breaking of the left-right symmetry at a high energy scale. Finally, such theories can also be embedded within an $S O(10)$ grand unified context and feature gauge coupling unification.

With a particle content exhibiting three extra vector bosons, three right-handed neutrinos, and several new scalar (Higgs) fields whose components possess a double, a single, or a vanishing electric charge, minimal left-right symmetric models (MLRSM) feature various collider signatures that can be used as probes for new physics [15-24,26]. The Higgs sector of the model in particular has recently been investigated [27-39]. In the present work, we study several Higgs processes giving rise to the production of four-lepton and three-lepton systems and focus on setups where these final states are stemming from the production and decay either of a pair of doubly charged Higgs bosons or of an associated pair composed of a doubly charged Higgs boson and a singly charged Higgs or vector boson. Unlike most earlier studies, we consider a framework where both type I and type II seesaw mechanisms are implemented and contribute to neutrino mass generation. Equivalently, the neutral component of the left-handed $\left(\Delta_{L}\right)$ and right-handed $\left(\Delta_{R}\right)$ Higgs triplets both acquire nonvanishing vacuum expectation values $v_{L, R} \neq 0$. This opens up certain decay modes that are forbidden when $v_{L}=0$ (as for a type I seesaw mechanism) and that could be used as handles on distinguishing a $v_{L}=0$ from a $v_{L} \neq 0$ scenario. While the phenomenology corresponding to the $v_{L} \neq 0$ case has been widely studied in a pure type II seesaw context [40-47], the one connected to a mixed type I/II seesaw model still remains to be comprehensively explored. Several processes become open only by virtue of the nonzero $v_{L}$ value, but still remain suppressed as $v_{L}$ is bound to be small. This nonvanishing $v_{L}$ value nevertheless allows us to weaken the constraints on the charged scalar particles stemming from flavor data.

Unlike in earlier MLRSM studies, we consider a high integrated luminosity of LHC collisions at a center-ofmass energy of $14 \mathrm{TeV}$. We adopt a scenario motivated by current experimental constraints (in particular on the doubly charged Higgs bosons) and perform a systematic comparison of expected new physics signals and Standard Model background including the simulation of the detector effects. The rest of this paper is organized as follows. In Sec. II, we discuss the MLRSM theoretical framework and its particle content, before designing a representative benchmark scenario that could be probed by multileptonic probes. In Sec. III, we study the collider phenomenology of this setup and quantitatively estimate how it could be discovered or constrained at the high-luminosity run of the LHC. We then generalize our findings as a function of the mass scale of the model, before concluding in Sec. IV.

\section{THE MINIMAL LEFT-RIGHT SYMMETRIC MODEL}

\section{A. Theoretical framework}

Left-right symmetric models [15-24] are Standard Model extensions where the gauge symmetry group is enlarged to $S U(3)_{c} \times S U(2)_{L} \times S U(2)_{R} \times U(1)_{B-L}$. In minimal leftright-symmetric incarnations, the theory is additionally invariant under discrete left-right symmetry (or $D$-parity) transformations that relate the $S U(2)_{L}$ and $S U(2)_{R}$ sectors. Focusing on the minimal model field content shown in Table I, the right-handed Standard Model fermionic degrees of freedom are grouped into $S U(2)_{R}$ doublets, which render the presence of right-handed neutrinos natural. Compared to the Standard Model case, the Higgs sector is significantly enriched. The Standard Model $S U(2)_{L}$ Higgs doublet is promoted to a Higgs $S U(2)_{L} \times S U(2)_{R}$ bidoublet $\Phi$ allowing one to write gauge-invariant Yukawa interactions yielding Dirac mass terms for all fermions, and the breaking of the gauge symmetry down to the electroweak symmetry further requires the presence of an $S U(2)_{R}$ triplet $\Delta_{R}$. In order to maintain the theory $D$-parity symmetric, we include its $S U(2)_{L}$ counterpart $\Delta_{L}$. All field transformations under a $D$-parity symmetry operation hence read [39]

$$
\left\{\begin{array} { l } 
{ q _ { L } \leftrightarrow q _ { R } } \\
{ \ell _ { L } \leftrightarrow \ell _ { R } } \\
{ \Delta _ { L } \leftrightarrow \Delta _ { R } } \\
{ \Phi \leftrightarrow \Phi ^ { \dagger } }
\end{array} \text { or } \quad \left\{\begin{array}{l}
q_{L} \leftrightarrow q_{R}^{c} \\
\ell_{L} \leftrightarrow \ell_{R}^{c} \\
\Delta_{L} \leftrightarrow \Delta_{R}^{*}, \\
\Phi \leftrightarrow \Phi^{t}
\end{array}\right.\right.
$$

depending whether $D$-parity symmetry is seen as a generalized parity or charge conjugation.

The Lagrangian of the model is written as $[48,49]$

$$
\mathcal{L}_{\text {MLRSM }}=\mathcal{L}_{\text {kinetic }}+\mathcal{L}_{\text {Yukawa }}-V_{\text {scalar }},
$$

TABLE I. MLRSM field content, presented together with the

\begin{tabular}{|c|c|c|c|c|}
\hline Field & $S U(3)$ & $S U(2)_{L}$ & $S U(2)_{R}$ & $U(1)_{B-L}$ \\
\hline$q_{L}=\left(\begin{array}{l}u_{L} \\
d_{L}\end{array}\right)$ & 3 & 2 & 1 & $\frac{1}{3}$ \\
\hline$q_{R}=\left(\begin{array}{l}u_{R} \\
d_{R}\end{array}\right)$ & 3 & 1 & 2 & $\frac{1}{3}$ \\
\hline$\ell_{L}=\left(\begin{array}{c}\nu_{L} \\
e_{L}\end{array}\right)$ & 1 & 2 & 1 & -1 \\
\hline$\ell_{R}=\left(\begin{array}{l}\nu_{R} \\
e_{R}\end{array}\right)$ & 1 & 1 & 2 & -1 \\
\hline$\Phi=\left(\begin{array}{ll}\phi_{1}^{0} & \phi_{1}^{+} \\
\phi_{2}^{-} & \phi_{2}^{0}\end{array}\right)$ & 1 & 2 & 2 & 0 \\
\hline$\Delta_{L}=\left(\begin{array}{cc}\Delta_{L}^{+} / \sqrt{2} & \Delta_{L}^{++} \\
\Delta_{L}^{0} & -\Delta_{L}^{+} / \sqrt{2}\end{array}\right)$ & 1 & 3 & 1 & 2 \\
\hline$\Delta_{R}=\left(\begin{array}{cc}\Delta_{R}^{+} / \sqrt{2} & \Delta_{R}^{++} \\
\Delta_{R}^{0} & -\Delta_{R}^{+} / \sqrt{2}\end{array}\right)$ & 1 & 1 & 3 & 2 \\
\hline
\end{tabular}
representations under $S U(3)_{c} \times S U(2)_{L} \times S U(2)_{R} \times U(1)_{B-L}$. 
where $\mathcal{L}_{\text {kinetic }}$ contains standard kinetic and gauge interaction terms for all fields. The Yukawa interactions read

$$
\mathcal{L}_{\text {Yukawa }}=-\left[y_{i j} \bar{\ell}_{i L} \Phi \ell_{j R}+y_{i j}^{\prime} \bar{\ell}_{i L} \tilde{\Phi} \ell_{j R}+Y_{i j} \bar{q}_{i L} \Phi q_{j R}+Y_{i j}^{\prime} \bar{q}_{i L} \tilde{\Phi} q_{j R}+\frac{1}{2} f_{i j}\left(\ell_{i R}^{c} \tilde{\Delta}_{R} \ell_{j R}+(R \leftrightarrow L)\right)+\text { H.c. }\right]
$$

where $\tilde{\Phi}=\sigma_{2} \Phi^{*} \sigma_{2}$ and $\tilde{\Delta}_{L, R}=i \sigma_{2} \Delta_{L, R}$. As a consequence of the built-in $D$-symmetry, both $S U(2)_{L}$ and $S U(2)_{R}$ neutrino couplings $f_{L}$ and $f_{R}$ are equal to a unique value $f$. The scalar potential $V_{\text {scalar }}$ is given by

$$
\begin{aligned}
V_{\text {scalar }}= & -\mu_{1}^{2} \operatorname{Tr}\left[\Phi^{\dagger} \Phi\right]-\mu_{2}^{2} \operatorname{Tr}\left[\Phi^{\dagger} \tilde{\Phi}+\tilde{\Phi}^{\dagger} \Phi\right]-\mu_{3}^{2} \operatorname{Tr}\left[\Delta_{L}^{\dagger} \Delta_{L}+\Delta_{R}^{\dagger} \Delta_{R}\right]+\lambda_{1}\left(\operatorname{Tr}\left[\Phi^{\dagger} \Phi\right]\right)^{2} \\
& +\lambda_{2}\left\{\left(\operatorname{Tr}\left[\Phi^{\dagger} \tilde{\Phi}\right]\right)^{2}+\left(\operatorname{Tr}\left[\tilde{\Phi}^{\dagger} \Phi\right]\right)^{2}\right\}+\lambda_{3} \operatorname{Tr}\left[\Phi^{\dagger} \tilde{\Phi}\right] \operatorname{Tr}\left[\tilde{\Phi}^{\dagger} \Phi\right]+\lambda_{4} \operatorname{Tr}\left[\Phi^{\dagger} \Phi\right] \operatorname{Tr}\left[\Phi^{\dagger} \tilde{\Phi}+\tilde{\Phi}^{\dagger} \Phi\right] \\
& +\rho_{1}\left\{\left(\operatorname{Tr}\left[\Delta_{L}^{\dagger} \Delta_{L}\right]\right)^{2}+\left(\operatorname{Tr}\left[\Delta_{R}^{\dagger} \Delta_{R}\right]\right)^{2}\right\}+\rho_{2}\left\{\operatorname{Tr}\left[\Delta_{L} \Delta_{L}\right] \operatorname{Tr}\left[\Delta_{L}^{\dagger} \Delta_{L}^{\dagger}\right]+\operatorname{Tr}\left[\Delta_{R} \Delta_{R}\right] \operatorname{Tr}\left[\Delta_{R}^{\dagger} \Delta_{R}^{\dagger}\right]\right\}+\rho_{3} \operatorname{Tr}\left[\Delta_{L}^{\dagger} \Delta_{L}\right] \operatorname{Tr}\left[\Delta_{R}^{\dagger} \Delta_{R}\right] \\
& +\rho_{4}\left\{\operatorname{Tr}\left[\Delta_{L} \Delta_{L}\right] \operatorname{Tr}\left[\Delta_{R}^{\dagger} \Delta_{R}^{\dagger}\right]+\operatorname{Tr}\left[\Delta_{L}^{\dagger} \Delta_{L}^{\dagger}\right] \operatorname{Tr}\left[\Delta_{R} \Delta_{R}\right]+\alpha_{1} \operatorname{Tr}\left[\Phi^{\dagger} \Phi\right] \operatorname{Tr}\left[\Delta_{L}^{\dagger} \Delta_{L}+\Delta_{R}^{\dagger} \Delta_{R}\right]\right. \\
& +\left\{\alpha_{2}\left(\operatorname{Tr}\left[\Phi^{\dagger} \tilde{\Phi}\right] \operatorname{Tr}\left[\Delta_{L}^{\dagger} \Delta_{L}\right]+\operatorname{Tr}\left[\tilde{\Phi}^{\dagger} \Phi\right] \operatorname{Tr}\left[\Delta_{R}^{\dagger} \Delta_{R}\right]\right)+\text { H.c. }\right\} \\
& +\alpha_{3} \operatorname{Tr}\left[\Phi \Phi^{\dagger} \Delta_{L} \Delta_{L}^{\dagger}+\Phi^{\dagger} \Phi \Delta_{R} \Delta_{R}^{\dagger}\right]+\beta_{1} \operatorname{Tr}\left[\Phi^{\dagger} \Delta_{L}^{\dagger} \Phi \Delta_{R}+\Delta_{R}^{\dagger} \Phi^{\dagger} \Delta_{L} \Phi\right]+\beta_{2} \operatorname{Tr}\left[\Phi^{\dagger} \Delta_{L}^{\dagger} \tilde{\Phi} \Delta_{R}+\Delta_{R}^{\dagger} \tilde{\Phi}^{\dagger} \Delta_{L} \Phi\right] \\
& +\beta_{3} \operatorname{Tr}\left[\tilde{\Phi}^{\dagger} \Delta_{L}^{\dagger} \Phi \Delta_{R}+\Delta_{R}^{\dagger} \Phi^{\dagger} \Delta_{L} \tilde{\Phi}\right],
\end{aligned}
$$

where we have introduced scalar mass parameters $\mu_{i}$ and quartic scalar interaction strengths $\lambda_{i}, \rho_{i}, \alpha_{i}$, and $\beta_{i}$.

The symmetry-breaking pattern is split into two steps,

$$
\begin{aligned}
& S U(2)_{L} \times S U(2)_{R} \times U(1)_{B-L} \\
& \quad \stackrel{\left\langle\Delta_{R}\right\rangle}{\longrightarrow} S U(2)_{L} \times U(1)_{Y} \stackrel{\langle\Phi\rangle}{\longrightarrow} U(1)_{\text {e.m. }} .
\end{aligned}
$$

At high energy, the $S U(2)_{L} \times S U(2)_{R} \times U(1)_{B-L}$ symmetry group is first spontaneously broken down to the electroweak symmetry group, and at a lower energy scale, the electroweak symmetry is further broken down to electromagnetism. The first breaking step results from the nonvanishing vacuum expectation value acquired by the neutral component of the $\Delta_{R}$ field at the minimum of the scalar potential, while electroweak symmetry breaking is induced by the vacuum expectation values of the neutral components of the Higgs bidoublet. Introducing the notations

$$
\left\langle\phi_{1,2}^{0}\right\rangle=\frac{k_{1,2}}{\sqrt{2}} \quad \text { and } \quad\left\langle\Delta_{L, R}^{0}\right\rangle=\frac{v_{L, R}}{\sqrt{2}},
$$

the Standard Model vacuum expectation value is given by $v_{\mathrm{SM}}=\sqrt{k_{1}^{2}+k_{2}^{2}} \approx 246 \mathrm{GeV}$. Without any loss of generality, we make use of a rotation in the $S U(2)_{L} \times S U(2)_{R}$ space so that only one of the neutral components of the Higgs bidoublet acquires a large vacuum expectation value, $k_{1} \approx v_{\mathrm{SM}}$ and $k_{2} \approx 0$. In addition, electroweak precision tests constrain $v_{L}$ to be smaller than $2 \mathrm{GeV}$ [3], and the breaking pattern of Eq. (2.5) enforces $v_{R}$ to be much greater than $k_{1}$. $D$-parity invariance moreover imposes the $g_{L}$ and $g_{R}$ gauge couplings to be equal to a common value $g$.

Under those assumptions, we neglect all contributions to the gauge boson masses that are proportional to $v_{L}$, so that these masses approximatively read

$$
\begin{aligned}
& M_{W}^{2}=\frac{g^{2}}{4} k_{1}^{2}, \quad M_{W_{R}}^{2}=\frac{g^{2}}{2} v_{R}^{2}, \\
& M_{Z}^{2}=\frac{g^{2} k_{1}^{2}}{4 \cos ^{2} \theta_{W}}\left(1-\frac{\cos ^{2} 2 \theta_{W}}{2 \cos ^{4} \theta_{W}} \frac{k_{1}^{2}}{v_{R}^{2}}\right), \quad M_{Z_{R}}^{2}=\frac{g^{2} v_{R}^{2} \cos ^{2} \theta_{W}}{\cos 2 \theta_{W}},
\end{aligned}
$$

with $\theta_{W}$ indicating the weak mixing angle.

After symmetry breaking, the Higgs sector is left with four neutral scalar fields $H_{0}^{0}, H_{1}^{0}, H_{2}^{0}$, and $H_{3}^{0}$, two neutral pseudoscalar fields $A_{1}^{0}$ and $A_{2}^{0}$ (as well as two neutral Goldstone bosons eaten by the $Z$ and $Z_{R}$ bosons), two singly charged Higgs bosons $H_{1}^{ \pm}$and $H_{2}^{ \pm}$(as well as four charged Goldstone bosons eaten by the $W^{ \pm}$and $W_{R}^{ \pm}$ bosons), and two doubly charged scalar bosons $H_{L}^{ \pm \pm}$and $H_{R}^{ \pm \pm}$. Within the above-mentioned approximations, the scalar masses are given by

$$
\begin{aligned}
& M_{H_{0}^{0}}^{2}=2 \lambda_{1} k_{1}^{2}, \quad M_{H_{1}^{0}}^{2}=\frac{1}{2} \alpha_{3} v_{R}^{2}, \quad M_{H_{2}^{0}}^{2}=2 \rho_{1} v_{R}^{2}, \\
& M_{H_{3}^{0}}^{2}=\frac{1}{2}\left(\rho_{3}-2 \rho_{1}\right) v_{R}^{2}, \\
& M_{A_{1}^{0}}^{2}=\frac{1}{2} \alpha_{3} v_{R}^{2}-2\left(2 \lambda_{2}-\lambda_{3}\right) k_{1}^{2}, \quad M_{A_{2}^{0}}^{2}=\frac{1}{2} v_{R}^{2}\left(\rho_{3}-2 \rho_{1}\right), \\
& M_{H_{1}^{ \pm}}^{2}=\frac{1}{2}\left(\rho_{3}-2 \rho_{1}\right) v_{R}^{2}+\frac{1}{4} \alpha_{3} k_{1}^{2}, \quad M_{H_{2}^{ \pm}}^{2}=\frac{1}{2} \alpha_{3} v_{R}^{2}+\frac{1}{4} \alpha_{3} k_{1}^{2}, \\
& M_{H_{L}^{ \pm \pm}}^{2}=\frac{1}{2}\left(\rho_{3}-2 \rho_{1}\right) v_{R}^{2}+\frac{1}{2} \alpha_{3} k_{1}^{2}, \quad M_{H_{R}^{ \pm \pm}}^{2}=2 \rho_{2} v_{R}^{2}+\frac{1}{2} \alpha_{3} k_{1}^{2} \text {, }
\end{aligned}
$$

the left-right triplet mixing induced by the $\beta_{i}$ potential terms being neglected as suppressed by the $k_{1}^{2} / v_{R}^{2}$ ratio, and the different states being not necessarily mass ordered. 
Turning to the neutrino sector, the $f_{i j}$ Yukawa couplings of Eq. (2.3) give rise to neutrino Majorana masses after symmetry breaking, as the neutral component of the $\Delta_{L}$ Higgs field acquires a nonzero vacuum expectation value [24],

$$
v_{L}=\frac{\beta_{2} k_{1}^{2}}{\left(2 \rho_{1}-\rho_{3}\right) v_{R}}
$$

which satisfies $\left|v_{L}\right| \ll v_{\mathrm{SM}} \ll\left|v_{R}\right|$. The $6 \times 6$ neutrino mass matrix is then given, in the $\left(\nu_{L}, \nu_{R}\right)$ gauge eigenbasis, by

$$
M=\left(\begin{array}{cc}
\sqrt{2} f v_{L} & M_{D} \\
M_{D}^{T} & M_{R}
\end{array}\right) .
$$

The light and heavy neutrino sectors are decoupled in the absence of any left-right mixing potentially induced by a nonvanishing Dirac mass matrix $M_{D}$, and the light and heavy masses are in this case respectively governed by the $f$ and $M_{R}$ parameters. In contrast, nonzero Dirac masses give rise to neutrino mixings parametrized by a mixing matrix $R^{\nu}$,

$R^{\nu}=\left(\begin{array}{cc}U & S \\ T & V\end{array}\right)=\left(\begin{array}{cc}1-\frac{1}{2} R R^{\dagger} & R \\ -R^{\dagger} & 1-\frac{1}{2} R^{\dagger} R\end{array}\right)\left(\begin{array}{cc}U_{L} & 0 \\ 0 & U_{R}\end{array}\right)$.

In this expression, $R=M_{D} M_{R}^{-1}$, while $U_{L}$ and $U_{R}$ are, respectively, the two matrices diagonalizing the light and heavy neutrino mass matrices $M_{\nu}$ and $M_{R}$,

$$
\begin{aligned}
& \left(M_{\nu}\right)_{i j}=\sqrt{2} f_{i j} v_{L}-\left(M_{D}\right)_{i k}\left(M_{R}\right)_{k l}^{-1}\left(M_{D}^{T}\right)_{l j} \quad \text { with } \\
& \left(M_{D}\right)_{i j}=\frac{1}{\sqrt{2}} y_{i j} k_{1} \quad \text { and } \quad\left(M_{R}\right)_{i j}=\sqrt{2} f_{i j} v_{R} .
\end{aligned}
$$

This shows that light neutrino masses arise from a combination of type I and type II seesaw contributions and are derived from the diagonalization of the upper-left block of the mass matrix $M$ by a $U_{L}$ rotation, $U_{L}$ being the usual Pontecorvo-Maki-Nakagawa-Sakata (PMNS) matrix.

\section{B. Constraints and MLRSM benchmark scenarios with light doubly charged Higgs bosons}

In order to design simplified phenomenologically viable benchmark scenarios for the collider studies performed in the next section, we account for various constraints arising from current data.

The scalar sector of the theory must include a neutral scalar boson that is consistent with the observation of a Standard-Model-like Higgs boson with a mass of about $125 \mathrm{GeV}$. We enforce the $H_{0}^{0}$ boson to be such a boson, its mass being set to

$$
M_{H_{0}^{0}}=125 \mathrm{GeV} .
$$

The extra neutral scalar bosons, in particular those with a large bidoublet component (i.e., $H_{1}^{0}$ and $A_{1}^{0}$ ), generally mediate tree-level flavor-changing neutral interactions. Consequently, their mass is constrained to be above about $10 \mathrm{TeV}$ [50-54] from experimental kaon mixing data [55]. On the other hand, the scalar potential perturbativity and unitarity further push these Higgs bosons to be heavier than $18 \mathrm{TeV}$. The minimum mass configuration is realized for a $W_{R}$ boson satisfying $M_{W_{R}}>8 \mathrm{TeV}$ [56], a constraint that is by far compatible with the most stringent LHC bounds regardless of the details of the right-handed neutrino sector [57-61]. Consequently, we impose

$$
\begin{aligned}
& M_{H_{1}^{0}}=M_{A_{1}^{0}}=20 \mathrm{TeV}, \\
& M_{W_{R}}=10 \mathrm{TeV} \Leftrightarrow v_{R}=21639.39 \mathrm{GeV} .
\end{aligned}
$$

Saturating the present limits on the $S U(2)_{L}$ triplet vacuum expectation value arising from the $\rho$-paramater [3],

$$
v_{L}=2 \mathrm{GeV},
$$

and recalling that we have chosen

$$
k_{1} \approx v_{\mathrm{SM}}=246 \mathrm{GeV} \quad \text { and } \quad k_{2} \approx 0,
$$

we make use of Eq. (2.8) together with the setup of Eqs. (2.13) and (2.14) to numerically derive the (tree-level) parameters of the scalar potential,

$\lambda_{1}=0.129, \quad \alpha_{3}=1.708, \quad 2 \lambda_{2}-\lambda_{3}=0$.

On the basis of the results of the ATLAS searches for samesign dileptonic new physics signals [62], we moreover impose a lower bound on the masses of the doubly charged scalars $H_{L}^{ \pm \pm}$and $H_{R}^{ \pm \pm}$. Assuming that the branching ratios into electronic and muonic final states are both equal to $50 \%$, the $S U(2)_{L}$ and $S U(2)_{R}$ doubly charged Higgs-boson masses have to be larger than $785 \mathrm{GeV}$ and $675 \mathrm{GeV}$, respectively. We adopt an optimistic scenario and take their masses close to the experimental limits,

$$
M_{H^{++}} \equiv M_{H_{L}^{++}}=M_{H_{R}^{++}}=800 \mathrm{GeV} .
$$

This leads to

$$
\rho_{2}=6.2817 \times 10^{-4}, \quad \rho_{3}-2 \rho_{1}=0.0025,
$$

with the other gauge and scalar boson masses being therefore

$$
\begin{aligned}
& M_{Z_{R}}=16754 \mathrm{GeV}, \quad M_{H_{2}^{0}}=6756 \mathrm{GeV}, \\
& M_{H_{3}^{0}}=M_{A_{2}^{0}}=767 \mathrm{GeV}, \quad M_{H_{1}^{+}}=784 \mathrm{GeV}, \\
& M_{H_{2}^{+}}=20 \mathrm{TeV} .
\end{aligned}
$$


The parameters related to the neutrino masses and mixings can be constrained by LHC searches in the samesign dilepton plus dijet channel, such a signature being relevant for probing right-handed neutrino production via an $s$-channel $W_{R}$ exchange [63]. A wide fraction of the parameter space turns out to be excluded by 8 and $13 \mathrm{TeV}$ LHC data due to the nonobservation of any such signal [64,65]. For the choice of Eq. (2.14), right-handed neutrinos have to be heavy. The neutrino sector can also undergo several low energy tests from intensity frontier experiments looking for lepton-number violation (such as neutrinoless double-beta decays) or for lepton-flavor violation (like rare muonic decays into electrons such as $\mu \rightarrow e \gamma$ or $\mu \rightarrow 3 e$ ). The associated combined limits induce a hierarchy between the mass of the $S U(2)_{R}$ scalar bosons and the mass of the heaviest right-handed neutrino that must be 2 to 10 times smaller $[66,67]$ for $M_{W_{R}}=3.5 \mathrm{TeV}$. These bounds are, however, derived under the assumption that either a type I or a type II seesaw mechanism is implemented. Considering a model featuring a combination of type I and type II seesaw mechanisms (as in this work) enables us to evade those bounds $[68,69]$, the $S U(2)_{R}$ triplet scalar masses even being allowed to be smaller than the heaviest right-handed neutrino mass. Right-handed neutrinos could nevertheless be indirectly constrained by neutrinoless double-beta decays and cosmology [70-72].

With the above assignments, the only parameters left to be considered are the mass parameters of the heavy and light neutrinos, $M_{N_{i}}$, with $i=1,2,3,4,5,6$. We shall consider the lighter degrees of freedom (i.e., the left-handed neutrinos corresponding to $i=1,2,3$ ) to have a mass of the order of $0.1 \mathrm{eV}$ to agree with cosmological data. For simplicity, we assume a unified scenario for the righthanded neutrino sector,

$$
M_{N_{4}}=M_{N_{5}}=M_{N_{6}} \equiv M_{N_{R}}=12 \mathrm{TeV},
$$

which allows one to evade all the above-mentioned bounds and feature perturbative Yukawa couplings of $\mathcal{O}(1)$.

In addition, the heavy-light neutrino mixing is constrained from neutrino oscillation data. After having fixed all physical masses of the neutrinos and assuming that $\left|f v_{L}\right| \ll\left|M_{D}\right| \ll\left|M_{R}\right|$, the $M_{R}$ mass matrix can be read off these masses, in a first approximation, and the type II seesaw contribution to the light neutrino masses can be deduced from the (input) $M_{\nu}$ matrix [73],

$$
M_{D}=M_{N_{R}}\left(\frac{v_{L}}{v_{R}}-\frac{M_{\nu}}{M_{N_{R}}}\right)^{1 / 2} .
$$

The Dirac mass contribution is of about $100 \mathrm{GeV}$ with an assumed $M_{\nu} \sim 0.1 \mathrm{eV}$ for the considered scenario. The cancellation in Eq. (2.22) between the type I and type II seesaw contributions are hence fine-tuned to the level of $10^{10}$, such a fine-tuning being stable against quantum corrections [74]. This further impacts the light-heavy neutrino mixing matrix,

$$
T=-R^{\dagger} U_{L}=-\left(M_{R}^{-1}\right)^{\dagger} M_{D}^{\dagger} U_{L} \approx 0.018 U_{L},
$$

which also dictates the strength of the heavy neutrino decays into left-handed leptons and a Standard Model $W$-boson. The rest of the neutrino mixing matrix stems from the unitarity properties of that matrix, which subsequently fixes the strength of the heavy neutrino decays into a final state system made of a right-handed lepton and a possibly off-shell heavy gauge boson. The heavy-light neutrino mixing is actually rather large, as $v_{L}$ is large, which opens the door for unusual heavy neutrino decays into the left-handed sector.

\section{Main feature of our benchmark scenario}

In order to determine the experimental signatures associated with the production of two scalar bosons or one vector and one scalar boson at the LHC, we first present, in Table II, the decay table related to the relevant (lighter) fields. The large right-handed neutrino masses have deep consequences on the couplings of the $S U(2)_{L}$ doubly charged and singly charged Higgs bosons to leptons, as they are proportional to $M_{N_{R}} / v_{R}$. This impacts the decay

TABLE II. Branching ratios (BR) associated with the different decay channels of the light scalar and vector bosons within the considered MLRSM realization. We independently denote by $\ell=e, \mu, \tau$ any lepton flavor, and we omit any channel whose branching ratio is smaller than $0.1 \%$.

\begin{tabular}{lr}
\hline \hline Doubly charged bosons & \\
\hline $\operatorname{BR}\left(H_{R}^{++} \rightarrow \ell^{+} \ell^{+}\right)$ & $33.3 \%$ \\
$\operatorname{BR}\left(H_{L}^{++} \rightarrow \ell^{+} \ell^{+}\right)$ & $32.39 \%$ \\
$\operatorname{BR}\left(H_{L}^{++} \rightarrow W^{+} \ell^{+} \nu_{\ell}\right)$ & $1.00 \%$ \\
\hline \hline Singly charged bosons & \\
\hline $\operatorname{BR}\left(H_{1}^{+} \rightarrow \ell^{+} \nu_{\ell}\right)$ & $33.26 \%$ \\
$\operatorname{BR}\left(H_{1}^{+} \rightarrow W^{+} Z\right)$ & $0.22 \%$ \\
$\operatorname{BR}\left(W_{R}^{+} \rightarrow q \bar{q}^{\prime}\right)$ & $100 \%$ \\
\hline \hline
\end{tabular}

Neutral bosons

\begin{tabular}{ll}
\hline $\operatorname{BR}\left(H_{3}^{0} \rightarrow \nu_{\ell} \nu_{\ell}\right)$ & $31.8 \%$ \\
$\operatorname{BR}\left(H_{3}^{0} \rightarrow Z \nu_{\ell} \nu_{\ell}\right)$ & $0.83 \%$ \\
$\operatorname{BR}\left(H_{3}^{0} \rightarrow W \ell \nu_{\ell}\right)$ & $0.38 \%$ \\
$\operatorname{BR}\left(H_{3}^{0} \rightarrow Z Z\right)$ & $0.52 \%$ \\
$\operatorname{BR}\left(H_{3}^{0} \rightarrow W W\right)$ & $0.26 \%$ \\
$\operatorname{BR}\left(A_{2}^{0} \rightarrow \nu_{\ell} \nu_{\ell}\right)$ & $32.1 \%$ \\
$\operatorname{BR}\left(A_{2}^{0} \rightarrow Z \nu_{\ell} \nu_{\ell}\right)$ & $0.85 \%$ \\
$\operatorname{BR}\left(A_{2}^{0} \rightarrow W \ell \nu_{\ell}\right)$ & $0.39 \%$ \\
\hline \hline
\end{tabular}



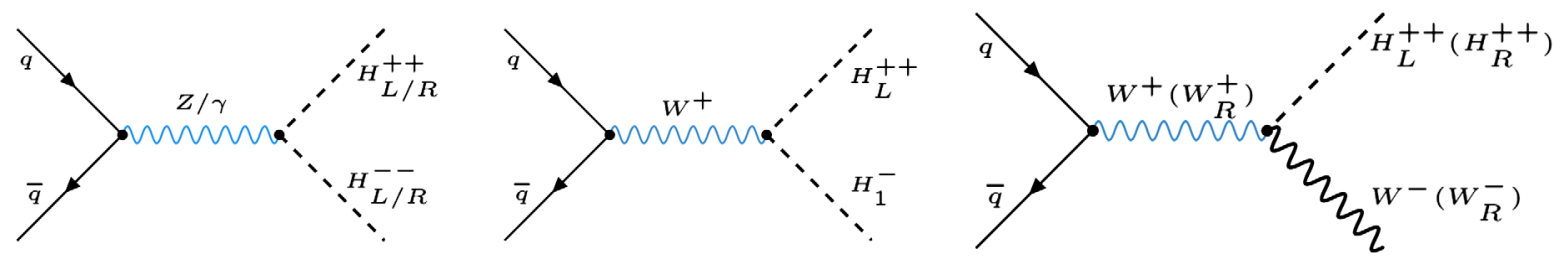

FIG. 1. Representative Feynman diagram of the various MLRSM processes giving rise to multileptonic final states.

pattern of the scalar fields which will rarely decay into nonleptonic final states. The $H_{L}^{ \pm \pm}$boson hence almost decays exclusively into a same-sign dileptonic system and the $H_{1}^{+}$Higgs boson into a lepton-neutrino pair. Other nonnegligibly small $H_{L}^{++}$decay modes include a virtual $H_{1}^{+}$ boson, and these sum up to a branching ratio of $3 \%$ after considering all three lepton flavors. The $S U(2)_{R}$ doubly charged $H_{R}^{++}$boson in contrast only decays into a samesign lepton pair, the potential decay modes into an $S U(2)_{R}$ gauge boson, and another scalar being kinematically closed. Finally, the lighter neutral $H_{3}^{0}$ and $A_{2}^{0}$ bosons almost exclusively decay invisibly, the neutral scalar boson $H_{3}^{0}$ nevertheless undergoing rare visible decays into electroweak gauge bosons, while the singly charged $W_{R}$ boson always decays into a dijet system.

As the considered new particles significantly decay into leptonic final states, natural collider probes include finalstate systems made of three or four leptons, as the corresponding Standard Model background is additionally small. As a consequence, we focus on the production of two doubly charged Higgs bosons and on the associated production of a doubly charged Higgs boson and a singly charged gauge or Higgs boson,

$$
p p \rightarrow H_{L / R}^{++} H_{L / R}^{--}, \quad p p \rightarrow H_{L}^{ \pm \pm} H_{1}^{\mp}, \quad \text { and } p p \rightarrow H_{R}^{ \pm \pm} W_{R}^{\mp},
$$

for which representative leading-order Feynman diagrams are shown in Fig. 1. Other hard-scattering processes involving new Higgs and gauge bosons could also possibly lead to multileptonic final states, but with suppressed and negligible rates. For instance, $H_{L}^{ \pm \pm} W^{\mp}$ production suffers from a strong $v_{L}$ suppression, and the large mass of the heavier Higgs bosons yields to a severe phase-space suppression for any process in which they could be produced.

Leading-order cross sections are given in the top panel of Table III for LHC proton-proton collisions at a center-ofmass energy $\sqrt{s}=14 \mathrm{TeV}$. The production of a pair of doubly charged Higgs bosons proceeds via a Drell-Yan-like process and the exchange of an $s$-channel neutral gauge or Higgs boson. By virtue of the smallness of the Standard Model Yukawa couplings and the heavy mass of the $Z_{R}$ and extra Higgs bosons, virtual $Z$-boson and photon contributions dominate and lead to a cross section of about 0.197 and $0.076 \mathrm{fb}$ for the production of a pair of $S U(2)_{L}$ and
$S U(2)_{R}$ doubly charged Higgs bosons, respectively, including in both cases a next-to-leading order (NLO) $K$-factor of 1.25 [75]. Doubly charged Higgs bosons can also be produced in association with a singly charged Higgs or gauge boson. While the $H_{R}^{ \pm \pm} W_{R}^{\mp}$ production cross section is negligible by virtue of the heavy mass of the $W_{R}$ boson, a $H_{L}^{ \pm \pm} H_{1}^{\mp}$ system can be produced via the exchange of a lighter Standard Model $W$-boson, other diagrams contributing to a smaller extent. The associated cross section is of about $0.38 \mathrm{fb}$.

Including the relevant branching ratios, four-lepton final states arise from the production and decay of a pair of doubly charged Higgs bosons each decaying into a samesign dilepton, the associated cross section being of about $0.12 \mathrm{fb}$. On the other hand, trileptonic final states originate from the associated production of a doubly charged and a singly charged Higgs boson, with a similar total rate of $0.17 \mathrm{fb}$. Other leptonic final states, such as those featuring a same-sign dilepton system, cannot be produced with a sufficiently large rate to be relevant. Despite the smallness of these cross sections, we demonstrate in Sec. III that the

TABLE III. Production cross sections associated with the set of processes shown in Eq. (2.24), for proton-proton collisions at a center-of-mass energy of $14 \mathrm{TeV}$ and in the context of the adopted benchmark scenario. The cross sections are obtained by multiplying the results returned by MG5_aMC [76], when leadingorder matrix elements are convoluted with the leading-order set of NNPDF 2.3 parton densities [77], with an NLO $K$-factor of 1.25 [75]. Total production rates are presented in the left panel, whereas branching ratios into the two final states of interest are included in the right panel. We independently denote by $\ell=e, \mu$ any light lepton flavor (and the lepton flavors can be different within any given process).

Total rate at $\sqrt{s}=14 \mathrm{TeV}$

\begin{tabular}{lr}
\hline$p p \rightarrow H_{L}^{++} H_{L}^{--}$ & \\
$p p \rightarrow H_{R}^{++} H_{R}^{--}$ & $0.197 \mathrm{fb}$ \\
$p p \rightarrow H_{L}^{++} H_{1}^{-}$ & $0.076 \mathrm{fb}$ \\
$p p \rightarrow H_{L}^{--} H_{1}^{+}$ & $0.28 \mathrm{fb}$ \\
\hline
\end{tabular}

Total rate at $\sqrt{s}=14 \mathrm{TeV}$

$p p \rightarrow H_{L / R}^{++} H_{L / R}^{--} \rightarrow \ell^{+} \ell^{+} \ell^{-} \ell^{-}$

$0.12 \mathrm{fb}$

$p p \rightarrow H_{L}^{ \pm \pm} H_{1}^{\mp} \rightarrow \ell^{ \pm} \ell^{ \pm} \ell^{\mp}+\mathscr{E}_{T}$

$0.17 \mathrm{fb}$ 
associated MLRSM signals can potentially be observed (or excluded) at the high-luminosity run of the LHC.

\section{LHC PHENOMENOLOGY}

For our analysis, we have used the FEYNRULES package [78] and the existing implementation of the MLRSM model [49] to generate a Universal FeynRules Output (UFO) model [79] that can be used within the MG5_aMC platform [76]. We have generated hard-scattering events both for the signal processes of Eq. (2.24) and for the Standard Model background, the tree-level matrix elements being convoluted with the leading-order set of NNPDF 2.3 parton distributions [77]. The simulation of the QCD environment (parton showering and hadronization) has been performed with PYTHIA 6 [80], and we have included the response of a CMS-like detector with DELPHES 3 [81] that internally relies on FASTJET [82] for the reconstruction of the physics objects, using the anti- $k_{T}$ algorithm with a radius parameter $R=0.4$ [83].

We require, at the matrix-element level, that all leptons and jets have a transverse momentum $p_{T}^{\text {gen }}$ and pseudorapidity $\eta^{\text {gen }}$ satisfying

$$
\begin{aligned}
p_{T}^{\text {gen }}(j) & >10 \mathrm{GeV}, & p_{T}^{\text {gen }}(\ell) & >10 \mathrm{GeV}, \\
\left|\eta^{\text {gen }}(j)\right| & <2.5, \quad \text { and } & \left|\eta^{\text {gen }}(\ell)\right| & <2.5,
\end{aligned}
$$

and are separated in the transverse plane by an angular distance of at least 0.4 ,

$$
\begin{aligned}
\Delta R^{\operatorname{gen}}(j, j) & >0.4, \quad \Delta R^{\operatorname{gen}}(j, \ell)>0.4, \quad \text { and } \\
\Delta R^{\operatorname{gen}}(\ell, \ell) & >0.4 .
\end{aligned}
$$

We analyze the reconstructed events with MADANALYSIS 5 [84] and impose a basic event preselection where the reconstructed leptons and jets are required to be central and to have a transverse momentum larger than $20 \mathrm{GeV}$,

$$
\begin{array}{ll}
p_{T}(j) & >20 \mathrm{GeV}, \quad p_{T}(\ell)>20 \mathrm{GeV}, \\
|\eta(j)| & <2.5, \quad \text { and } \quad|\eta(\ell)|<2.5 .
\end{array}
$$

We furthermore ignore any lepton lying within a cone of radius $R=0.4$ centered on a jet.

\section{A. Four-lepton probes}

After simulating all the potential contributions to the Standard Model background (except for fake and chargemisidentificaton contributions), our preselection implies that the main background to a four-lepton signal are events issued from the production of a pair of (possibly off-shell) $Z$-bosons where both weak bosons decay leptonically. Subdominant contributions are expected to originate from the production of a $W W Z$ system and the associated production of a top quark-antiquark pair with a Z-boson. These last components of the background could potentially be rejected by (at least loosely) vetoing the presence of

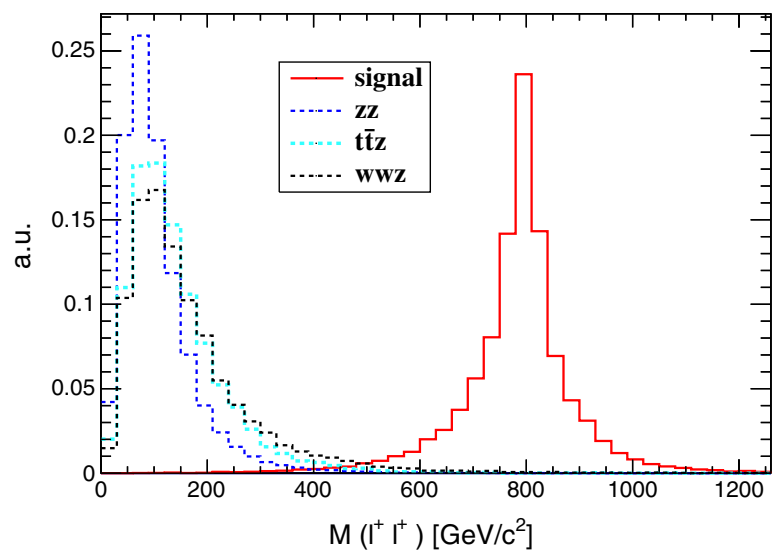

FIG. 2. Normalized invariant mass spectrum of the system made of the two positively charged leptons, after selecting events containing two pairs of same-sign leptons and vetoing the presence of $b$-tagged jets for the signal and the background.

missing energy and $b$-tagged jets in the final state. The signal fiducial cross section, normalized at the NLO accuracy and including the basic preselection is of $0.11 \mathrm{fb}$, for a corresponding background cross section of $18.9 \mathrm{fb}, 1.3 \mathrm{fb}$, and $0.13 \mathrm{fb}$ for the $Z$-boson pair, $t \bar{t} Z$, and tribosonic components, respectively. These last numbers include a QCD next-tonext-to-leading-order (NNLO) $K$-factor of 1.72 for the diboson case [85] and NLO ones of 1.38 and 1.04 for the two other processes [76,86], respectively.

To optimize the signal significance, we select events featuring exactly two pairs of opposite-charge leptons and veto those exhibiting any $b$-tagged jet. Signal leptons originating from the decay of heavy Higgs bosons, we further impose that the $p_{T}$ of the three leading leptons $p_{T}\left(\ell_{1}\right), p_{T}\left(\ell_{2}\right)$, and $p_{T}\left(\ell_{3}\right)$ satisfy

$p_{T}\left(\ell_{1}\right)>200 \mathrm{GeV}, \quad p_{T}\left(\ell_{2}\right)>150 \mathrm{GeV}, \quad$ and

$p_{T}\left(\ell_{3}\right)>60 \mathrm{GeV}$.

We then use, as an extra handle on the new physics signal, the invariant masses of the systems formed by the two pairs of same-sign leptons $M\left(\ell^{+} \ell^{+}\right)$and $M\left(\ell^{-} \ell^{-}\right)$. As in any resonance search, the shape of the signal spectrum is expected to show peaks corresponding to the physical masses of the parent particles, i.e., the doubly charged Higgs bosons in our case, as illustrated in Fig. 2. By imposing that these invariant masses fulfill

$M\left(\ell^{+} \ell^{+}\right)>300 \mathrm{GeV}$ and $M\left(\ell^{-} \ell^{-}\right)>300 \mathrm{GeV}$,

we are able to make the selection almost free from any background contamination, with about 15 background events being expected for a luminosity of $1 \mathrm{ab}^{-1}$.

In the left panel of Fig. 3, we present the sensitivity of the LHC to the MLRSM four-leptonic signal for different luminosity goals, the sensitivity $s$ (expressed in $\sigma$ ) being defined by [87] 

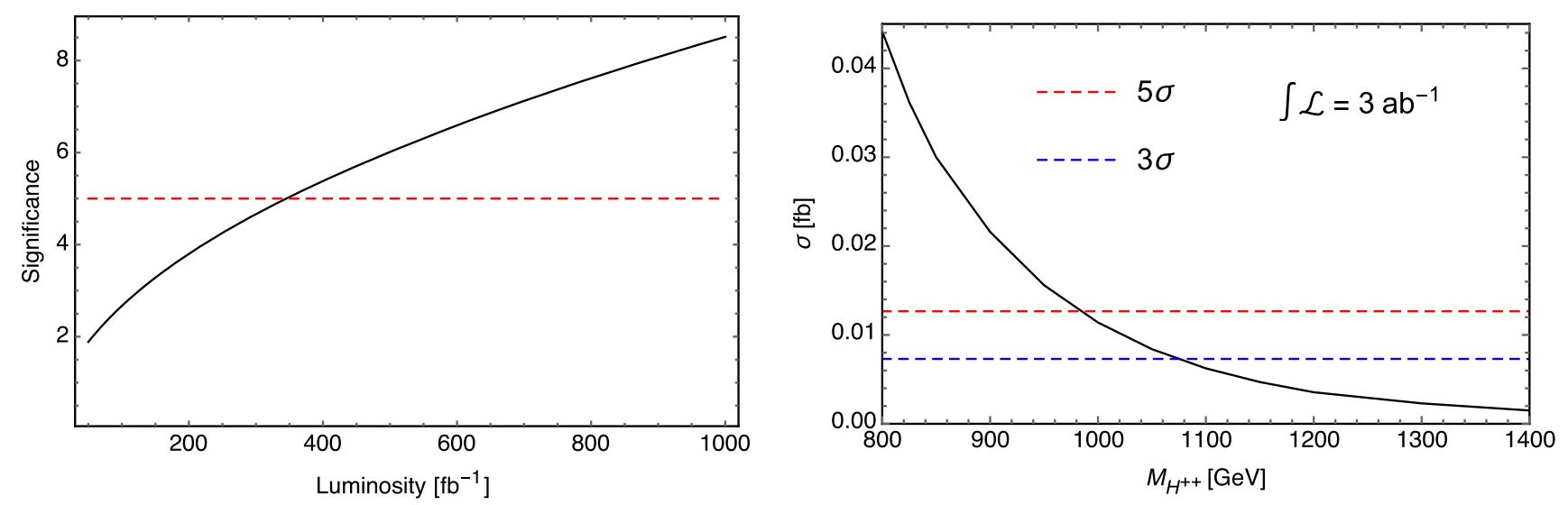

FIG. 3. Left: Dependence of the LHC sensitivity to the four-leptonic MLRSM signal on the integrated luminosity. Right: Dependence of the signal fiducial cross section after all selections on the double-charged Higgs boson mass $M_{H^{++}}$. The corresponding $3 \sigma$ (blue) and $5 \sigma$ (red) reference lines are indicated, assuming an integrated luminosity of $3 \mathrm{ab}^{-1}$.

$$
\begin{aligned}
s= & \sqrt{2} \times\left((S+B) \ln \left[\frac{(S+B)\left(B+x^{2}\right)}{B^{2}+(S+B) x^{2}}\right]\right. \\
& \left.-\frac{B^{2}}{x^{2}} \ln \left[1+\frac{x^{2} S}{B\left(B+x^{2}\right)}\right]\right)^{\frac{1}{2}},
\end{aligned}
$$

where $S$ and $B$, respectively, indicate the number of surviving signal and background events, and $x$ represents the systematic uncertainties on the background. Assuming $x=0.1 B$, a discovery could occur for about $400 \mathrm{fb}^{-1}$, while a signal significance of $s=8.5 \sigma$ could be expected for $1 \mathrm{ab}^{-1}$. Conversely, assuming that exclusion statements could be achieved for a sensitivity of about $2 \sigma$, the considered benchmark scenario could be excluded at the very beginning of the LHC Run 3. In order to assess the stability of our predictions with respect to the systematics, we vary the $x$ parameter to up to $20 \%$ of the background and investigate the induced modifications on the predictions. The latter are found robust and almost agnostic of such a change. In the right panel of Fig. 3, we generalize our conclusions to heavier scenarios and present the dependence of the fiducial cross section associated with the production of the four-lepton signal on the mass of the doubly charged Higgs boson. We compare it to the cross sections yielding potential $3 \sigma$ (blue line) and $5 \sigma$ (red line) observations for an integrated luminosity of $3 \mathrm{ab}^{-1}$. While the discovery reach can in principle be pushed above the 1.1 TeV regime, it is clear that hints for new physics could be observed for much heavier scales. Four-leptonic probes thus consist of key MLRSM signals, both by virtue of the associated background-free environment and by the moderate value of the signal cross sections.

\section{B. Trileptonic probes}

With a larger signal cross section, trileptonic probes are expected to provide good handles on any potential new physics signal. In our case, trileptonic signal events originate from the associated production of a doubly charged and a singly charged scalar. The signal production rate after preselection is of about $0.14 \mathrm{fb}$. Such a signal rate must compete with diboson and triboson backgrounds as well as with top backgrounds through the associated production of a top-antitop pair with a weak boson, any other background contribution having been found negligible after requesting the presence of three leptons. The corresponding cross sections are of 214.5, 32.5, 1, 2.77, and $1.71 \mathrm{fb}$ for $W Z, Z Z, W W W, t t Z$, and $t t W$ production, after including NNLO QCD $K$-factors in the diboson cases $\left(K_{W Z}=2.01\right.$ and $\left.K_{Z Z}=1.72[85,88]\right)$, an NLO QCD and electroweak $K$-factor in the triboson case (we have conservatively chosen $K_{W W W}=2.27$ [89], which differs from the much smaller $K$-factor of Ref. [90]), an NLO QCD $K$-factor in the $t t Z$ case $\left(K_{t t Z}=1.38\right.$ [86]), and an NLO $K$-factor including the resummation of the threshold logarithms in the $t t W$ case $\left(K_{t t W}=1.07\right.$ [91]).

Our selection requires events to contain two leptons carrying the same electric charge and a third lepton with an opposite charge. We moreover veto the presence of any reconstructed $b$-tagged jet to control the top-quark-induced background. As in the previous section, we impose a stringent selection of the lepton properties and constrain the transverse momentum of the two leading leptons to satisfy

$$
p_{T}\left(\ell_{1}\right)>250 \mathrm{GeV} \text { and } p_{T}\left(\ell_{2}\right)>80 \mathrm{GeV} \text {. }
$$

The signal selection efficiency is practically $100 \%$, while the dominant diboson background is reduced by a factor of 50. We then reconstruct the invariant mass of the samesign dilepton system and use it as an extra handle on the signal, requiring

$$
M_{\ell^{ \pm} \ell^{ \pm}}>300 \mathrm{GeV}
$$



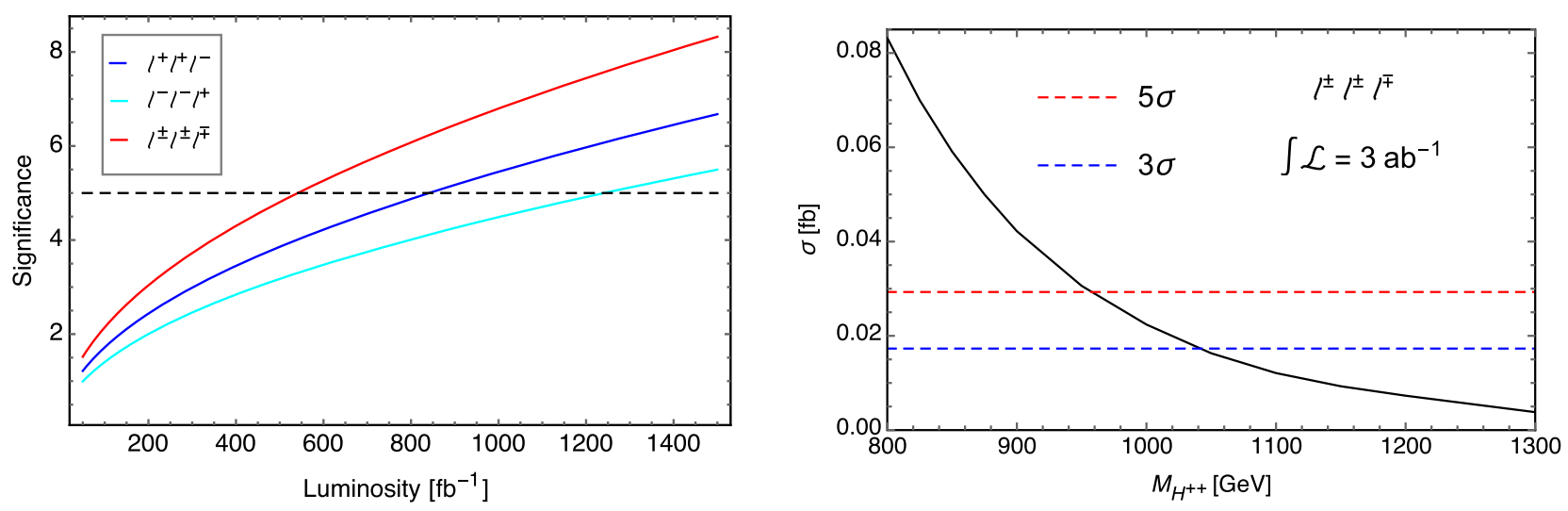

FIG. 4. Left: Dependence of the LHC sensitivity to the trileptonc MLRSM signal on the integrated luminosity. We separately indicate results for the $\ell^{+} \ell^{+} \ell^{-}$(blue line) and $\ell^{-} \ell^{-} \ell^{+}$(cyan line) channels, as well as for their combination (red line). Right: Dependence of the signal fiducial cross section after all selections on the double-charged Higgs boson mass $M_{H^{++}}$. The corresponding $3 \sigma$ (blue) and $5 \sigma$ (red) reference lines are indicated, assuming an integrated luminosity of $3 \mathrm{ab}^{-1}$.

which reduces the triboson and remaining top-induced background to a barely visible level. Although a veto on events featuring an opposite-sign dilepton system compatible with a $Z$-boson could help in reducing the remaining background, we instead require the selected events to contain a large amount of missing transverse energy,

$$
\mathbb{E}_{T}>150 \mathrm{GeV}
$$

These selections are sufficient to get a decent sensitivity to the signal, as shown in the left panel of Fig. 4 in which we present the dependence of the significance $s$ calculated as in Eq. (3.6) on the integrated luminosity. Both the $\ell^{+} \ell^{+} \ell^{-}$and $\ell^{-} \ell^{-} \ell^{+}$channels are expected to yield promising results, a $5 \sigma$ discovery being reachable within about $800-1200 \mathrm{fb}^{-1}$ of proton-proton collisions at $\sqrt{s}=$ $14 \mathrm{TeV}$ in both cases. Combining the two channels, a signal may even be observed during the earlier phase of the LHC Run 3, with a luminosity of about $500 \mathrm{fb}^{-1}$. While promising, the trilepton channel is not as competitive as the four-lepton one. Nevertheless, the option of a combination is conceivable and could potentially lead to an even better expectation. Our results are generalized in the heavier mass scale case on the right panel of Fig. 4, in which we present the dependence of the trilepton fiducial cross section (including thus the selection efficiency) on the mass of the doubly charged Higgs boson. In contrast to the fourleptonic channel, the entire luminosity expected to be collected during the high-luminosity run of the LHC will only allow us to barely reach the $\mathrm{TeV}$ mass regime.

\section{SUMMARY AND CONCLUSIONS}

Left-right symmetric models offer natural explanations for parity violation at the electroweak scale and an elegant way to address neutrino masses through the embedding of a seesaw mechanism. As a consequence of their symmetry breaking pattern, they feature additional scalar fields including $S U(2)_{L}$ and $S U(2)_{R}$ triplets. Such an enriched scalar sector offers various handles for discovering leftright symmetric new physics and for distinguishing it from other extensions of the Standard Model. In this work, we have focused on the multileptonic collider signatures of this scalar sector and estimated how the LHC could be sensitive to it in the upcoming years. By carefully designing first a benchmark configuration viable relatively to present data, we have found that the production of four-lepton and trilepton systems is enhanced and could be used as the main discovery mode of the model, even if current constraints already push the new physics masses to a high scale.

For our study, we have considered a scenario with doubly charged scalar masses fixed to $800 \mathrm{GeV}$, heavy righthanded neutrinos with masses of $12 \mathrm{TeV}$, and a $W_{R}$ boson of $10 \mathrm{TeV}$. With all other model parameters set to guarantee consistency with flavor data, we obtain a scenario featuring one light singly charged and two light neutral scalar bosons with large triplet components. Although the cross sections associated with the production of these light doubly charged and singly charged states have been found to be about $0.1-1 \mathrm{fb}$, we have shown, by relying on state-ofthe-art Monte Carlo simulations, that a simple selection strategy could allow for observing the resulting fourleptonic and trileptonic signals within the reach of the high-luminosity phase of the LHC. In other words, MLRSM singly charged and doubly charged Higgs bosons lying in the $\mathrm{TeV}$ range could be reached in a not too far future thanks to an analysis strategy yielding an almost background-free environment.

\section{ACKNOWLEDGMENTS}

P.P. and D.G. acknowledge support from the DSTSERB, India, project Grant No. EMR/2015/000333, and 
from the DST-FIST Grant No. SR/FST/PSIl-020/2009 for offering the computing resources needed by this work. B. F. has been partly supported by French state funds managed by the Agence Nationale de la Recherche (ANR) in the context of the LABEX ILP (ANR-11-IDEX-0004-02, ANR-10-LABX-63).
[1] G. Aad et al. (ATLAS Collaboration), Phys. Lett. B 716, 1 (2012).

[2] S. Chatrchyan et al. (CMS Collaboration), Phys. Lett. B 716, 30 (2012).

[3] K. A. Olive et al. (Particle Data Group), Chin. Phys. C 38, 090001 (2014).

[4] S. Weinberg, Phys. Rev. Lett. 43, 1566 (1979).

[5] P. Minkowski, Phys. Lett. 67B, 421 (1977).

[6] M. Gell-Mann, P. Ramond, and R. Slansky, Conf. Proc. C790927, 315 (1979).

[7] T. Yanagida, Conf. Proc. C7902131, 95 (1979).

[8] R. N. Mohapatra and G. Senjanovic, Phys. Rev. Lett. 44, 912 (1980).

[9] J. Schechter and J. W. F. Valle, Phys. Rev. D 22, 2227 (1980).

[10] R. N. Mohapatra and G. Senjanovic, Phys. Rev. D 23, 165 (1981).

[11] G. Lazarides, Q. Shafi, and C. Wetterich, Nucl. Phys. B181, 287 (1981).

[12] C. Wetterich, Nucl. Phys. B187, 343 (1981).

[13] J. Schechter and J. W. F. Valle, Phys. Rev. D 25, 774 (1982).

[14] R. Foot, H. Lew, X. G. He, and G. C. Joshi, Z. Phys. C 44, 441 (1989).

[15] J. C. Pati and A. Salam, Phys. Rev. D 10, 275 (1974); 11, 703(E) (1975).

[16] R. N. Mohapatra and J. C. Pati, Phys. Rev. D 11, 566 (1975).

[17] R. N. Mohapatra and J. C. Pati, Phys. Rev. D 11, 2558 (1975).

[18] G. Senjanovic and R. N. Mohapatra, Phys. Rev. D 12, 1502 (1975).

[19] R. N. Mohapatra, F. E. Paige, and D. P. Sidhu, Phys. Rev. D 17, 2462 (1978).

[20] G. Senjanovic, Nucl. Phys. B153, 334 (1979).

[21] R. N. Mohapatra and R. E. Marshak, Phys. Rev. Lett. 44, 1316 (1980); 44, 1643(E) (1980).

[22] C. S. Lim and T. Inami, Prog. Theor. Phys. 67, 1569 (1982).

[23] J. F. Gunion, J. Grifols, A. Mendez, B. Kayser, and F. I. Olness, Phys. Rev. D 40, 1546 (1989).

[24] N. G. Deshpande, J. F. Gunion, B. Kayser, and F. I. Olness, Phys. Rev. D 44, 837 (1991).

[25] P. Fileviez Perez, J. High Energy Phys. 03 (2009) 142.

[26] G. Senjanović and V. Tello, Phys. Rev. Lett. 119, 201803 (2017).

[27] G. Barenboim, M. Gorbahn, U. Nierste, and M. Raidal, Phys. Rev. D 65, 095003 (2002).

[28] J. Polak and M. Zralek, Phys. Lett. B 276, 492 (1992).

[29] G. Azuelos, K. Benslama, and J. Ferland, J. Phys. G 32, 73 (2006).

[30] D.-W. Jung and K. Y. Lee, Phys. Rev. D 78, 015022 (2008).
[31] G. Bambhaniya, J. Chakrabortty, J. Gluza, M. Kordiaczyńska, and R. Szafron, J. High Energy Phys. 05 (2014) 033.

[32] A. Alloul, M. Frank, B. Fuks, and M. Rausch de Traubenberg, Phys. Rev. D 88, 075004 (2013).

[33] B. Dutta, R. Eusebi, Y. Gao, T. Ghosh, and T. Kamon, Phys. Rev. D 90, 055015 (2014).

[34] G. Bambhaniya, J. Chakrabortty, J. Gluza, T. Jeliński, and M. Kordiaczynska, Phys. Rev. D 90, 095003 (2014).

[35] A. Maiezza, M. Nemevšek, and F. Nesti, Phys. Rev. Lett. 115, 081802 (2015).

[36] G. Bambhaniya, J. Chakrabortty, J. Gluza, T. Jelinski, and R. Szafron, Phys. Rev. D 92, 015016 (2015).

[37] P. S. B. Dev, R. N. Mohapatra, and Y. Zhang, J. High Energy Phys. 05 (2016) 174.

[38] K. S. Babu and S. Jana, Phys. Rev. D 95, 055020 (2017).

[39] A. Maiezza, G. Senjanović, and J. C. Vasquez, Phys. Rev. D 95, 095004 (2017).

[40] T. Han, B. Mukhopadhyaya, Z. Si, and K. Wang, Phys. Rev. D 76, 075013 (2007).

[41] P. Fileviez Perez, T. Han, G.-y. Huang, T. Li, and K. Wang, Phys. Rev. D 78, 015018 (2008).

[42] F. del Aguila and J. A. Aguilar-Saavedra, Nucl. Phys. B813, 22 (2009).

[43] A. G. Akeroyd, C.-W. Chiang, and N. Gaur, J. High Energy Phys. 11 (2010) 005.

[44] E. J. Chun, K. Y. Lee, and S. C. Park, Phys. Lett. B 566, 142 (2003).

[45] Z.-L. Han, R. Ding, and Y. Liao, Phys. Rev. D 91, 093006 (2015).

[46] Z.-L. Han, R. Ding, and Y. Liao, Phys. Rev. D 92, 033014 (2015).

[47] M. Chabab, M. C. Peyranere, and L. Rahili, Phys. Rev. D 90, 035026 (2014).

[48] A. Alloul, J. D'Hondt, K. De Causmaecker, B. Fuks, and M. Rausch de Traubenberg, Eur. Phys. J. C 73, 2325 (2013).

[49] A. Roitgrund, G. Eilam, and S. Bar-Shalom, Comput. Phys. Commun. 203, 18 (2016).

[50] G. Ecker, W. Grimus, and H. Neufeld, Phys. Lett. 127B, 365 (1983); 132B, 467(E) (1983).

[51] R. N. Mohapatra, G. Senjanovic, and M. D. Tran, Phys. Rev. D 28, 546 (1983).

[52] M. E. Pospelov, Phys. Rev. D 56, 259 (1997).

[53] Y. Zhang, H. An, X. Ji, and R. N. Mohapatra, Nucl. Phys. B802, 247 (2008).

[54] A. Maiezza, M. Nemevsek, F. Nesti, and G. Senjanovic, Phys. Rev. D 82, 055022 (2010).

[55] G. Beall, M. Bander, and A. Soni, Phys. Rev. Lett. 48, 848 (1982).

[56] A. Maiezza, M. Nemevšek, and F. Nesti, Phys. Rev. D 94, 035008 (2016). 
[57] M. Aaboud et al. (ATLAS Collaboration), Eur. Phys. J. C 78, 401 (2018).

[58] M. Aaboud et al. (ATLAS Collaboration), Phys. Rev. D 96, 052004 (2017).

[59] A. M. Sirunyan et al. (CMS Collaboration), Phys. Lett. B 769, 520 (2017); 772, 882(E) (2017).

[60] V. Khachatryan et al. (CMS Collaboration), Phys. Lett. B 770, 278 (2017).

[61] A. M. Sirunyan et al. (CMS Collaboration), J. High Energy Phys. 06 (2018) 128.

[62] M. Aaboud et al. (ATLAS Collaboration), Eur. Phys. J. C 78, 199 (2018).

[63] W.-Y. Keung and G. Senjanovic, Phys. Rev. Lett. 50, 1427 (1983).

[64] L. Basso, B. Fuks, M. E. Krauss, and W. Porod, J. High Energy Phys. 07 (2015) 147.

[65] A. M. Sirunyan et al. (CMS Collaboration), J. High Energy Phys. 05 (2018) 148.

[66] V. Tello, M. Nemevsek, F. Nesti, G. Senjanovic, and F. Vissani, Phys. Rev. Lett. 106, 151801 (2011).

[67] G. Bambhaniya, P. S. B. Dev, S. Goswami, and M. Mitra, J. High Energy Phys. 04 (2016) 046.

[68] D. Borah and A. Dasgupta, J. High Energy Phys. 11 (2015) 208.

[69] D. Borah and A. Dasgupta, J. High Energy Phys. 07 (2016) 022.

[70] D. Borah, Phys. Rev. D 94, 075024 (2016).

[71] M. Frank, B. Fuks, K. Huitu, S. K. Rai, and H. Waltari, J. High Energy Phys. 05 (2017) 015.

[72] J. Y. Araz, M. Frank, and B. Fuks, Phys. Rev. D 96, 015017 (2017).

[73] M. Nemevsek, G. Senjanovic, and V. Tello, Phys. Rev. Lett. 110, 151802 (2013).

[74] M. J. Luo and Q. Y. Liu, J. High Energy Phys. 12 (2008) 061.

[75] M. Muhlleitner and M. Spira, Phys. Rev. D 68, 117701 (2003).
[76] J. Alwall, R. Frederix, S. Frixione, V. Hirschi, F. Maltoni, O. Mattelaer, H. S. Shao, T. Stelzer, P. Torrielli, and M. Zaro, J. High Energy Phys. 07 (2014) 079.

[77] R. D. Ball et al., Nucl. Phys. B867, 244 (2013).

[78] A. Alloul, N. D. Christensen, C. Degrande, C. Duhr, and B. Fuks, Comput. Phys. Commun. 185, 2250 (2014).

[79] C. Degrande, C. Duhr, B. Fuks, D. Grellscheid, O. Mattelaer, and T. Reiter, Comput. Phys. Commun. 183, 1201 (2012).

[80] T. Sjostrand, S. Mrenna, and P. Z. Skands, J. High Energy Phys. 05 (2006) 026.

[81] J. de Favereau, C. Delaere, P. Demin, A. Giammanco, V. Lemaître, A. Mertens, and M. Selvaggi (DELPHES 3 Collaboration), J. High Energy Phys. 02 (2014) 057.

[82] M. Cacciari, G. P. Salam, and G. Soyez, Eur. Phys. J. C 72, 1896 (2012).

[83] M. Cacciari, G. P. Salam, and G. Soyez, J. High Energy Phys. 04 (2008) 063.

[84] E. Conte, B. Fuks, and G. Serret, Comput. Phys. Commun. 184, 222 (2013).

[85] F. Cascioli, T. Gehrmann, M. Grazzini, S. Kallweit, P. Maierhöfer, A. von Manteuffel, S. Pozzorini, D. Rathlev, L. Tancredi, and E. Weihs, Phys. Lett. B 735, 311 (2014).

[86] A. Kardos, Z. Trocsanyi, and C. Papadopoulos, Phys. Rev. D 85, 054015 (2012).

[87] G. Cowan, K. Cranmer, E. Gross, and O. Vitells, Eur. Phys. J. C 71, 1554 (2011); 73, 2501(E) (2013).

[88] M. Grazzini, S. Kallweit, D. Rathlev, and M. Wiesemann, Phys. Lett. B 761, 179 (2016).

[89] S. Yong-Bai, Z. Ren-You, M. Wen-Gan, L. Xiao-Zhou, and G. Lei, Phys. Rev. D 95, 073005 (2017).

[90] F. Campanario, V. Hankele, C. Oleari, S. Prestel, and D. Zeppenfeld, Phys. Rev. D 78, 094012 (2008).

[91] H. T. Li, C. S. Li, and S. A. Li, Phys. Rev. D 90, 094009 (2014). 